\title{
Research Paper \\ Comparison of Serum Levels of Hormone Omentin-1 and Insulin Resistance Markers in Active-Obese, Inactive-Obese and Inactive Normal-Weight Men
}

\section{Saeed Khosravi-Nejad ${ }^{1}$ (D) ${ }^{*}$ Mahmoud Nikseresht $^{1}$ (D)}

1. Department of Exercise Physiology, Faculty of Humanities, Ilam Branch, Islamic Azad University, Ilam, Iran.

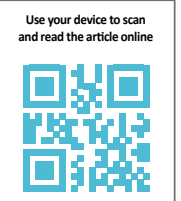

Citation Khosravi-Nejad S, Nikseresht M. Comparison of Serum Levels of Hormone Omentin-1 and Insulin Resistance Markers in Active-Obese, Inactive-Obese and Inactive Normal-Weight Men. The Journal of Qazvin University of Medical Sciences. 2019; 22(6):116-125. https://doi.org/10.32598/JQUMS.22.6.116

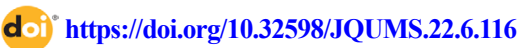

Received: 28 Aug 2018

Accepted: 19 Dec 2018

Available Online: 01 Feb 2019
Keywords:

Adipokine, Body

Mass Index, Insulin, Physical activity, Omentin-1

\begin{abstract}
Background Omentin-1 is an adipokine, mainly produced by visceral adipose tissue that improves insulin resistance.

Objective This study compared the serum levels of omentin-1 and insulin resistance markers in activeobese, inactive-obese and inactive normal-weight men.

Methods In this cross-sectional study, 45 men (35.0 44.2 years, no symptoms of illness) were assigned to active-obese $\left(n=16\right.$, Body Mass Index $(B M I)=28-33.9 \mathrm{~kg} / \mathrm{m}^{2}$ and $3-5$ score in the Physical Activity level questionnaire (PA-R score), inactive-obese $\left(n=18, B M I=28-33.9 \mathrm{~kg} / \mathrm{m}^{2}\right.$ and 1 in PA-R score) and inactive normal-weight ( $n=11, \mathrm{BMl}=18.5-24.9 \mathrm{~kg} / \mathrm{m}^{2}$ and 1 in PA-R score) groups. Blood samples were taken from all subjects in fasting state to measure the serum levels of omentin-1, insulin and glucose. Also, Homeostasis Model Assessment Index (HOMA-IR) for insulin resistance was assessed.

Findings The results showed that the omentin-1 concentration was significantly lower in inactive normalweight compared to the obese groups $(P<0.05)$. In the active-obese group, the glucose, insulin concentrations and insulin resistance index were similar to the inactive normal-weight group; however, these values were significantly lower than the inactive-obese group $(P<0.05)$. No significant difference was found between normal-inactive and obese-inactive groups for glucose concentration ( $P=0.079)$.

Conclusion Based on these findings, it can be said that physical activity is more effective than obesity. Thus, the obese men can improve the negative effects of obesity on insulin resistance markers by performing physical activity and is not required to reduce the size of obesity.
\end{abstract}

\section{Extended Abstract}

\section{Introduction}

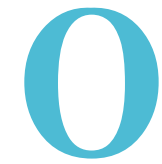

besity is associated with cardiovascular, kidney and lung diseases, type 2 diabetes and cancers [1]. Regular physical activity, in addition to weight control reduce the risk of such diseases [2]. Omentin-1 is an adi- pokine related to obesity; it plays a key role in controlling blood pressure, cardiovascular diseases and metabolic disorders [3]. The serum level of omentin-1 in inactive obese men is significantly higher than that of their healthy counterparts [4].

On the contrary, the plasma level of omentin-1 in obese men is lower than that of healthy weight men [5]. Nikseresht reported that insulin resistance index and insulin and glucose levels in obese men were significantly

\section{* Corresponding Author:}

Mahmoud Nikseresht, PhD.

Address: Department of Exercise Physiology, Faculty of Humanities, Ilam Branch, Islamic Azad University, Ilam, Iran.

Tel: +98 (84) 32227526

E-Mail: nikserasht@gmail.com 
higher than those of lean men [6]. Khoo et al. argued that an exercise-induced weight loss program has been more effective in improving insulin resistance, compared to dieting alone [7]. The current study compared the serum level of omentin- 1 and insulin resistance markers in active-obese, inactive-obese, and inactive healthy-weight men.

\section{Methods and Materials}

In this cross-sectional study, 45 healthy men were assigned into the following groups: 1 . Active-obese ( $\mathrm{n}=16$, Body Mass Index $(\mathrm{BMI})=28-33.9 \mathrm{~kg} / \mathrm{m}^{2}$, Physical Activity Rating (PAR) scores=3-5); 2. Inactive-obese $(n=18, B M I=28-33.9 \mathrm{~kg} /$ $\mathrm{m}^{2}$, PA-R score $\left.=1\right)$, and inactive-healthy $(\mathrm{n}=11, \mathrm{BMI}=18.5$ $24.9 \mathrm{~kg} / \mathrm{m}^{2}$, PA-R score=1) groups.

Fasting blood samples were obtained from all subjects to measure biochemical variables. Elisa test kits were used for measuring the concentrations of omentin-1 (EASTBIOPHARM, China) and insulin (Q-1-DIAPLUS, USA); for glucose, the glucose oxidase method (Glucose B-Test Kit, Wako Pure Chemical, Japan) was used. Furthermore, Homeostasis Model Assessment (HOMA-IR) was applied for measuring insulin resistance. Moreover, Beta cells ( $\beta$-cells) function (\%) was obtained according to the following formula:

Insulin resistance $=($ Fasting insulin in $\mathrm{mIU} / \mathrm{mL} \times$ Fasting glucose in $\mathrm{mmol} / \mathrm{L}) / 22.5$

$\beta$-cell function $=($ Fasting insulin in $\mathrm{mIU} / \mathrm{mL} \times 20) /($ Fasting glucose in $\mathrm{mmol} / \mathrm{L}-3.5$ )
One-way Analysis of Variance (ANOVA), Kruskal-Wallis test, and Gabriel's post hoc test were used for analyzing the obtained data. The significance level was set at $\mathrm{P}<0.05$.

\section{Results}

Kruskal-Wallis test results suggested that the concentration of omentin-1 in the inactive-healthy group was significantly lower, compared to the obese groups (Figure 1). One-way ANOVA results reported that insulin concentration and insulin resistance index in the active-obese group were similar to those of inactive-healthy group; these values were significantly lower than those of inactive-obese group. Glucose concentration was significantly lower that of the inactiveobese group only in the active-obese group. Furthermore, the achieved results revealed no significant difference between the groups in terms of $\beta$-cell function (Table 1).

\section{Conclusion}

In the present study, the serum level of omentin-1 was higher in obese groups, compared to the controls. Contrary to this finding, Ouerghi et al. reported that the plasma level of omentin- 1 in obese men is lower than that of healthy weight men [5]. This inconsistency may be because of the difference in the age of studied subjects. In their study, the mean age of subjects was 18 years which is considerably lower that of the present study subjects. Moreover, according to de Souza et al. the serum level of omentin-1 in lean subjects was higher than that of overweight and obese samples [10]. In their study, age (24-73 y) and BMI $\left(21-66 \mathrm{~kg} / \mathrm{m}^{2}\right)$ of the subjects were higher, compared to our study; this could explain the existing inconsistency.

Table 1. The anthropometric characteristics of study groups (Mean \pm SD)

\begin{tabular}{|c|c|c|c|c|c|c|c|}
\hline \multirow{2}{*}{ Variable } & \multirow{2}{*}{$\begin{array}{l}\text { Inactive Normal } \\
\text { Men }(n=11)\end{array}$} & \multirow{2}{*}{$\begin{array}{l}\text { Inactive Obese } \\
\text { Men (n=18) }\end{array}$} & \multirow{2}{*}{$\begin{array}{c}\text { Active Obese } \\
\text { Men (n=16) }\end{array}$} & \multirow{2}{*}{ ANOVA } & \multicolumn{3}{|c|}{ Gabriel's Post Hoc Test } \\
\hline & & & & & $P_{1}$ & $\mathbf{P}_{2}$ & $\mathbf{P}_{3}$ \\
\hline Age, y & $33.6 \pm 3.2$ & $36.3 \pm 5.2$ & $34.4 \pm 3.9$ & 0.314 & - & - & - \\
\hline Weight, kg & $68.8 \pm 8.9$ & $94.86 \pm 8.5$ & $87.4 \pm 8$ & $0.0006^{*}$ & $0.0007^{*}$ & $0.0009 *$ & 0.097 \\
\hline $\mathrm{BMI}, \mathrm{kg} / \mathrm{m}^{2}$ & $21.9 \pm 2$ & $30.6 \pm 1.9$ & $29.1 \pm 2.6$ & $0.0001^{*}$ & $0.0001^{*}$ & $0.0004^{*}$ & 0.282 \\
\hline Glucose, $\mathrm{mmol} / \mathrm{L}$ & $5.4 \pm 0.65$ & $5.9 \pm 0.42$ & $5.4 \pm 0.28$ & $0.003^{*}$ & 0.079 & 0.835 & $0.0001^{*}$ \\
\hline Insulin, mIU/mL & $5.4 \pm 1.5$ & $8.1 \pm 2.1$ & $4.6 \pm 1.9$ & $0.0001^{*}$ & $0.003^{*}$ & 0.707 & $0.0004 *$ \\
\hline Insulin resistance index & $1.34 \pm 0.51$ & $2.13 \pm 0.54$ & $1.12 \pm 0.44$ & $0.0003^{*}$ & $0.001 *$ & 0.607 & $0.0007^{*}$ \\
\hline (\%) $\beta$-cell function & $58.3 \pm 7.5$ & $68.4 \pm 24.5$ & $51.4 \pm 28$ & 0.137 & - & - & - \\
\hline
\end{tabular}

$\mathrm{P}_{1}$ : Difference between inactive normal and inactive obese groups; $\mathrm{P}_{2}$ : Difference between inactive normal and active obese groups; $\mathrm{P}_{3}$ : Difference between inactive obese and active obese groups; $* \mathrm{P}<0.05$ 


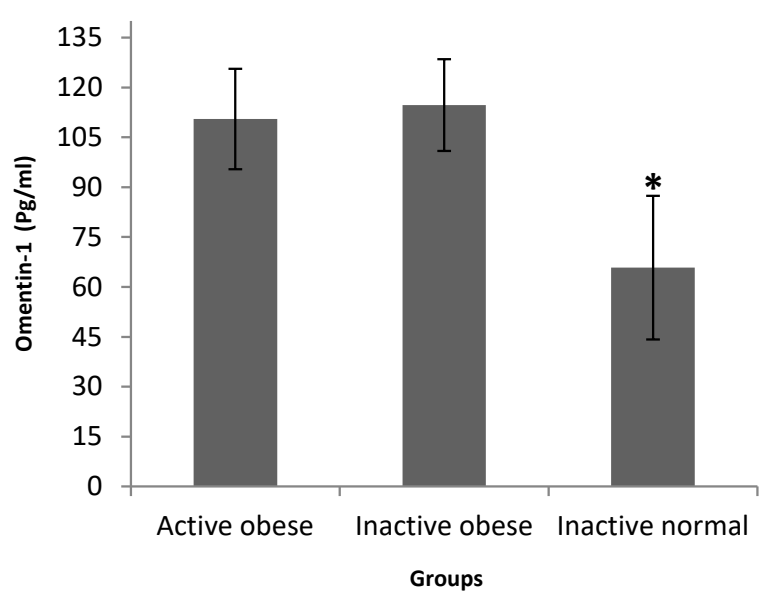

Figure 1. Omentin-1 serum level $(\mathrm{Pg} / \mathrm{mL})$ in the study groups

* Significant difference compared to other groups.

The current study reported that insulin concentration and insulin resistance index in the active-obese group were similar to those of inactive-healthy group. Moreover, these indices were significantly lower than those of the inactive-obese group. This result is consistent with the findings of Ouerghi et al. and Nikseresht who reported that the serum level of insulin in inactive obese men was significantly higher than lean men $[5,6]$. Moreover, Kadoglou et al. concluded that insulin resistance index in active patients was significantly lower than that of inactive patients [11].

In our study, glucose concentration in the active-obese and inactive-healthy groups was also similar; it was significantly lower than the inactive-obese group only in the active-obese group. Consistent with this result, Nikseresht [6] suggested that the serum level of glucose in inactive obese men was significantly higher than that of lean men. Overall, based on the current study, physical activity improves the status of insulin resistance markers without reducing obesity.

\section{Ethical Considerations}

\section{Compliance with ethical guidelines}

This study was registered with ethics code 36ECRIES and IRCT2012120411670N1 at the Clinical Trials Center Iran.

\section{Funding}

The present paper was extracted from the MSc. thesis of the first author, in Department of Exercise Physiology, Faculty of Humanities, Ilam Branch, Islamic Azad University.

\section{Authors' contributions}

Conceptualization, methodology, resources, writing-review, editing, supervision: Mahmoud Nikseresht; Writing original draft: Saeed Khosravi-Nejad; and Investigation: Mahmoud Nikseresht, Saeed Khosravi-Nejad.

\section{Conflict of interest}

The authors declared no conflict of interest. 


\title{
مقايسه سطح سرمى هورمون امنتين - ا و نشانكًان مقاومت به انسولين در مردان خاق فعال، خاق غيرفعال ونرمال غيرفعال
}

\author{
سعيد خسروىنزاد' هـ. "محمود نيكسرشت' (ه)
}

1- كروه فيزيولوزيى ورزشى، دانشكده علوم انسانى، واحد ايلام، دانشكاه آزاد اسلامى، ايلام، ايران.

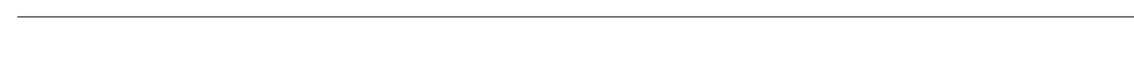

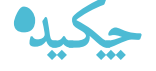

مينه امنتين-ا، آدييوكاينى است كه بيشتر در بافت جربى احشايى توليد و موجب بهبود حساسيت انسولين مي ششود.

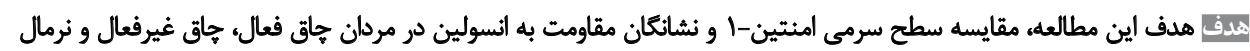
عيرفعال بود.

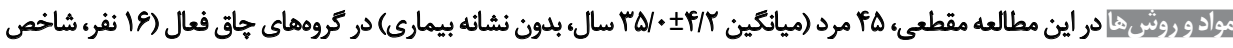

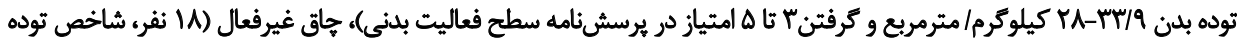

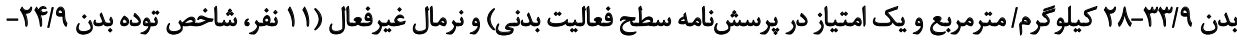

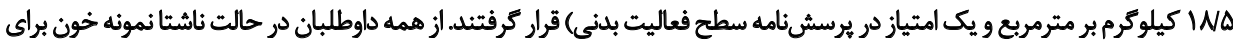

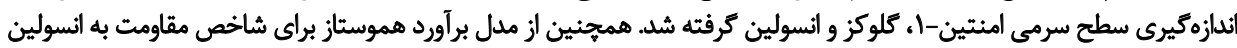
الستفاده شد.

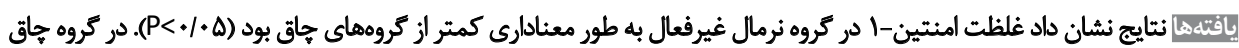

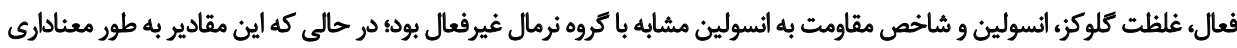

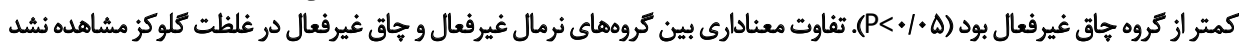
.$(P=\cdot / \cdot \vee q)$

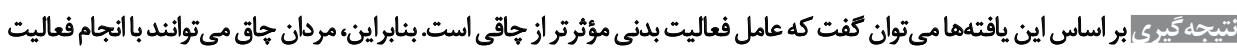

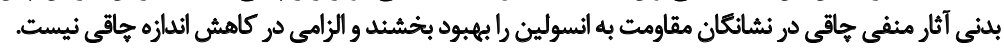

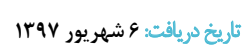

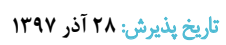
تاريخ ايتشار: TI بهمن
\end{abstract}

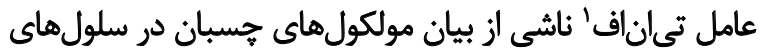

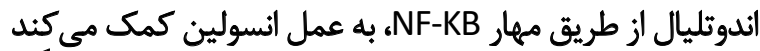

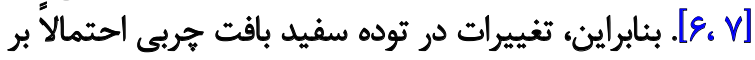

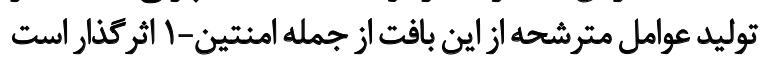

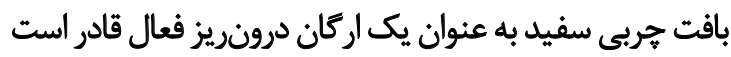

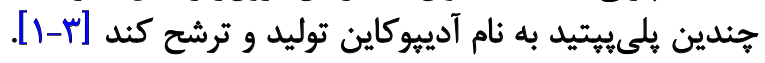

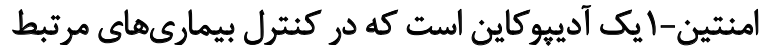

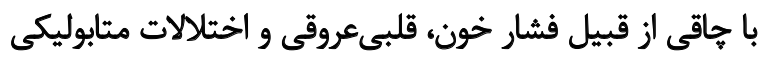
جاقى با مشكلات زيادى از جمله بيمارىهاى قلبىعروقى،

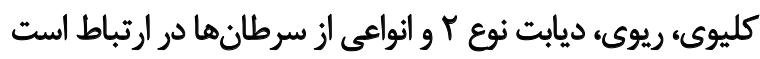

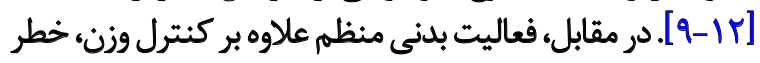

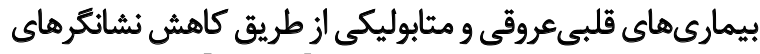

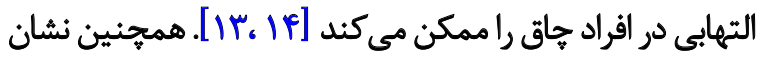

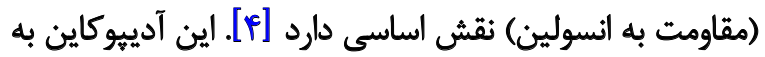

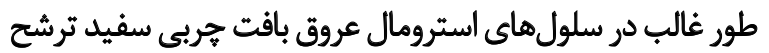

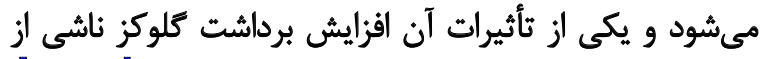

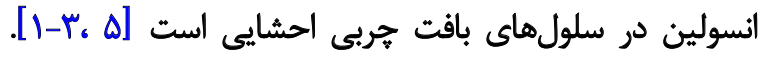

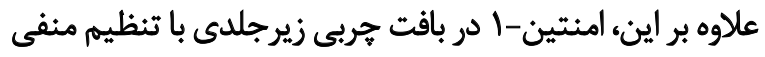
1. Tumor Necrosis Factor-alpha (TNF- $\alpha$ ) 


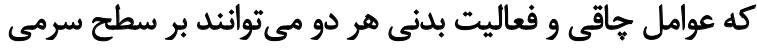

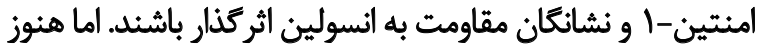

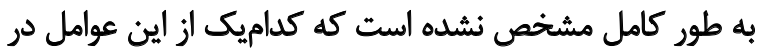

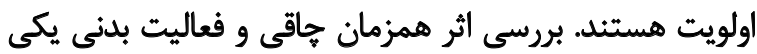

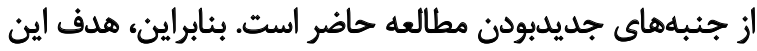

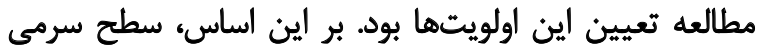

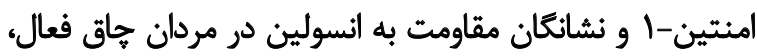
جاق غيرفعال و نرمال غيرفعال مقايسه شد.

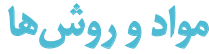

در اين مطالعه مقطعى، جاقى و فعاليت بدنى متغيرهاى

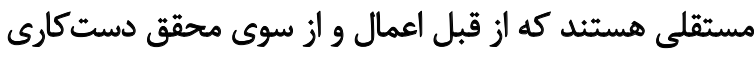

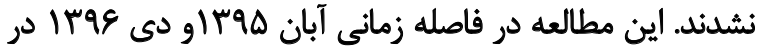

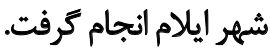

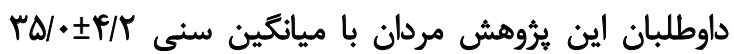

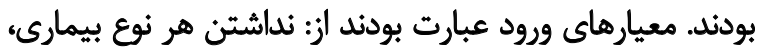

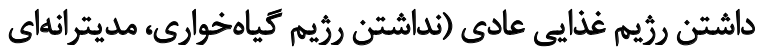

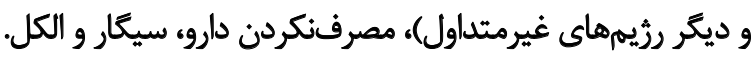

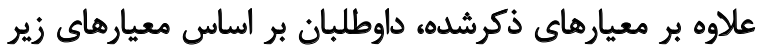

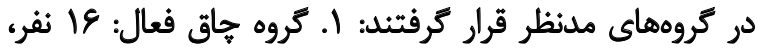

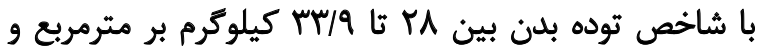

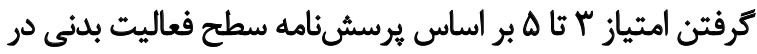

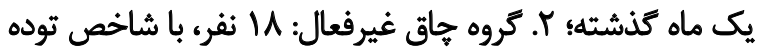

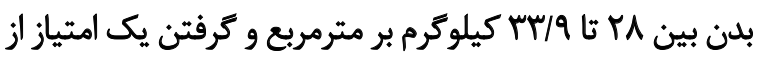

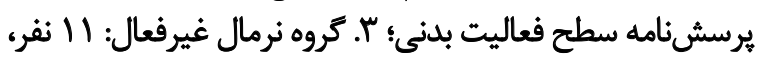

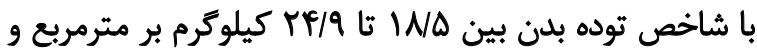
كرفتن يك امتياز از يرسشنامه سطح فئ فاليت بدنى.
داده شد كه التهاب مزمن درجه خفيف با افزايش فعاليت بدنى

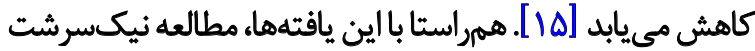
نشان داد شاخص مقاومت به انسولين و سطح سرمي انسابل انسولين

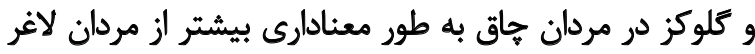

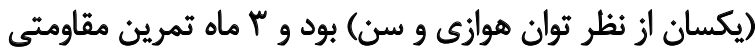

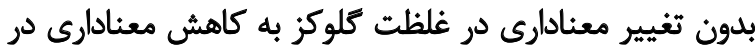

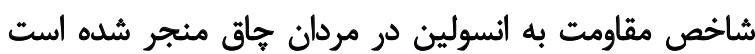

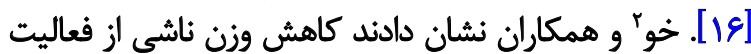

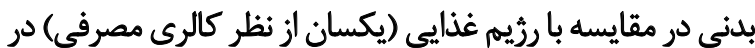

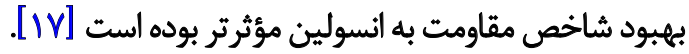

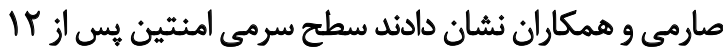

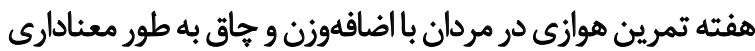

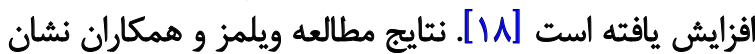

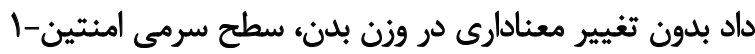

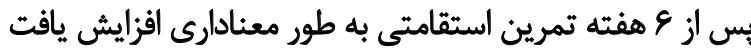

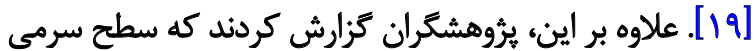

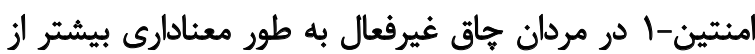

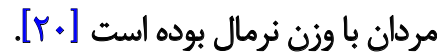

نتايج بررسىها درباره ياسخ امنتين-1 به هاقي و فعاليت

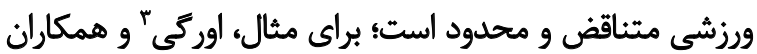

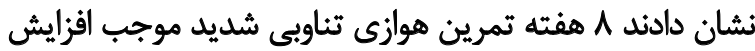

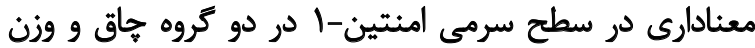

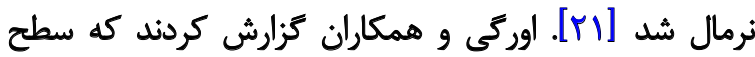

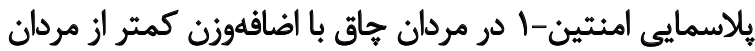

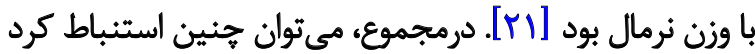

\section{Khoo}

3. Ouerghi

جدول (. يرسشنامه سطح فعاليت بدنى

\begin{tabular}{|c|c|}
\hline ميزان فعاليت بدنى & امتياز \\
\hline الجتناب از يبيادةووى يا فعاليت ورزشى (براى مثال: استفاده هميشكى از آسائسور، تا حد امكان استفاده از ماشين به جاى ييادهروى) & صفر \\
\hline 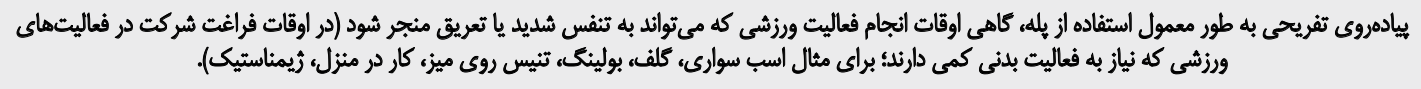 & 1 \\
\hline داشتن •ا أتا •و دقيقه فعاليت بدنى منظم در هفته & r \\
\hline 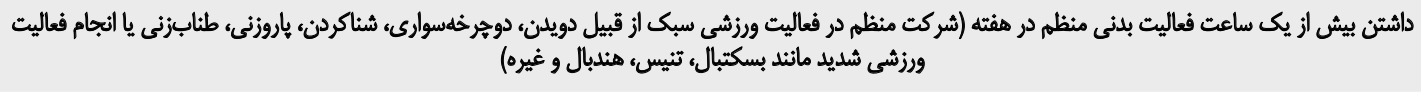 & r \\
\hline 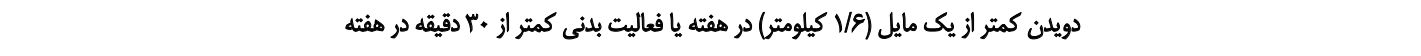 & r \\
\hline 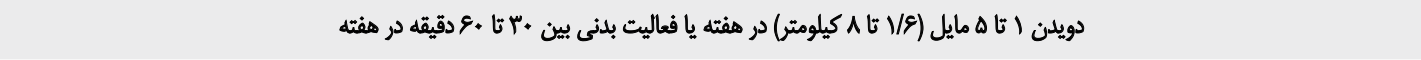 & $\Delta$ \\
\hline 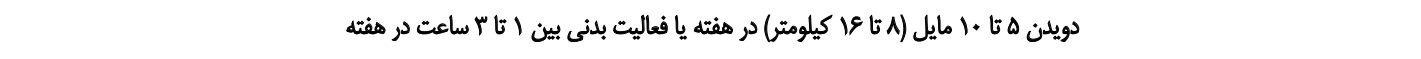 & 8 \\
\hline دويلن بيش از • ا عايل (وا كيلومتر) در هفته يا داشتن فعاليت بلنى بيش از ب ساعت در هفته & $\checkmark$ \\
\hline
\end{tabular}


طبق فرمول زير مقادير آن محاسبه شد. افزون بر اين، عملكرد

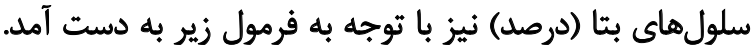

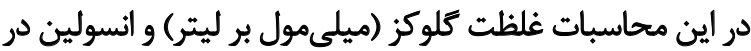

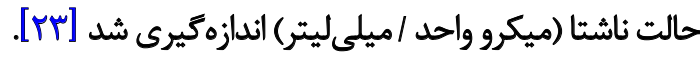
شاخص مقاومت انسولين=(غلظت كلوكز ناشتاءغلظت انسولين

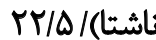

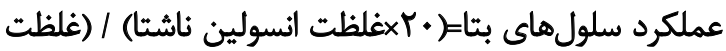

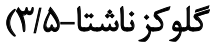

قد داوطلبان (به سانتىمتر) و وزن بدن آنها (به كيلوكرم) با

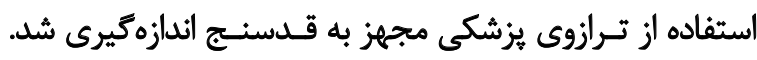

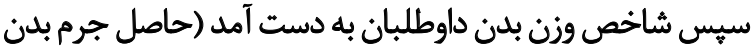
بر حسب كيلوكرم تقسيم بر مجذور قد بر حسب متر). دادهها براساس ميانكين ثلانحراف معيار تزارش شده است.

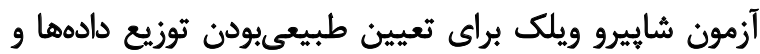

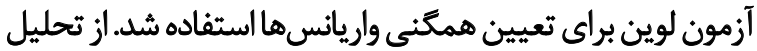

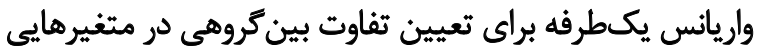

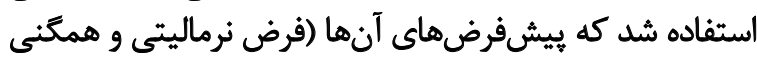

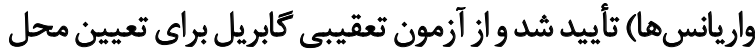

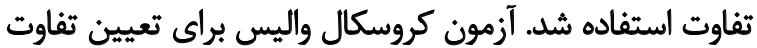

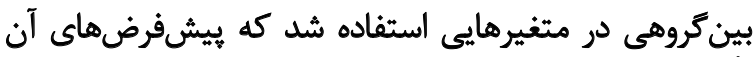

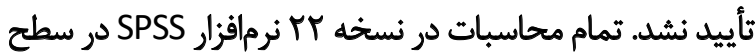

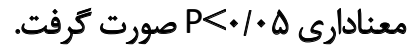

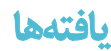

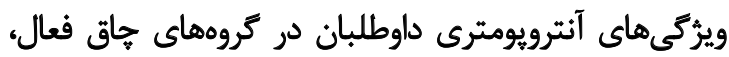

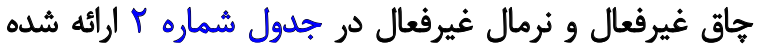

سطح فعاليت بدنى داوطلبان در كروههاى غيرفعال مشابه بود

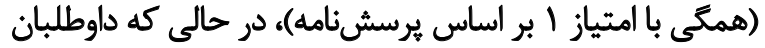

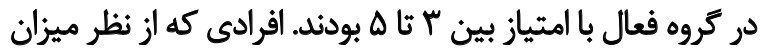

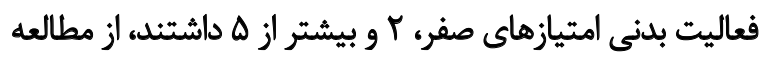

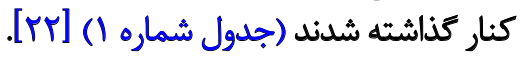
براى اندازهيرى سطح سرمى انسولين، كلوكز و امنتين-ا در

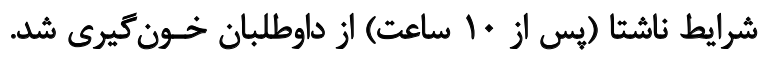

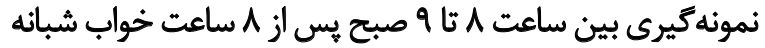

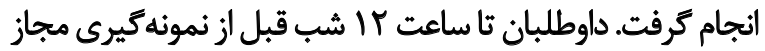

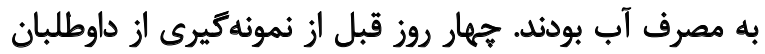

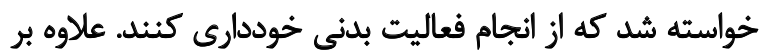

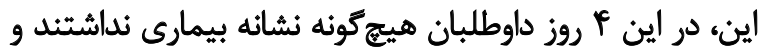

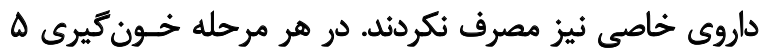

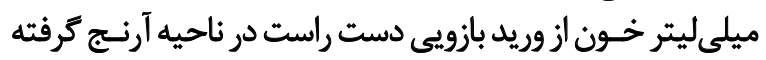

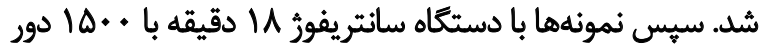
در دقيقه سانتريفوز و ســرم آنها جــــا شد.

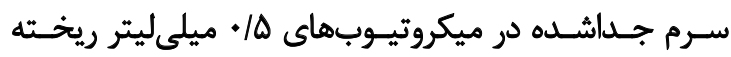

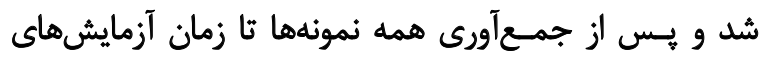

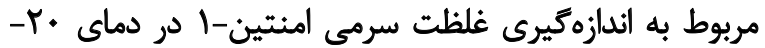

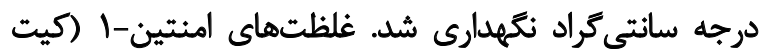
EASTBIOPHARM

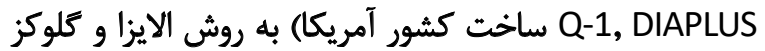

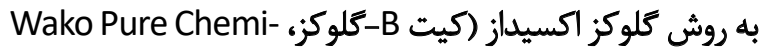

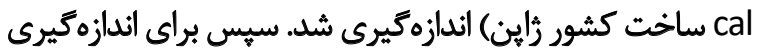
شاخص مقاومت انسولين از روش HOMA-IR

4. Homeostasis Model Assessment Ratio

جدول r. ويزّكى هاى آنترويومترى داوطلبان در كروهانى بررسى شده (ميانكينثانحراف معيار)

\begin{tabular}{|c|c|c|c|c|c|c|c|}
\hline \multicolumn{3}{|c|}{ تعقيبى (كابريل) } & \multirow{2}{*}{ ANOVA } & \multirow{2}{*}{ جاّ (1) نقفر) } & \multirow{2}{*}{ 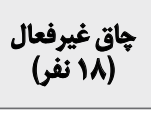 } & \multirow{2}{*}{ 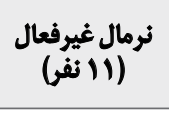 } & \multirow{2}{*}{ مثغيرها } \\
\hline $\mathbf{P}_{3}$ & $\mathbf{P}_{2}$ & $P_{1}$ & & & & & \\
\hline- & - & - &.$M I F$ & $M \in / F \pm m / q$ & $r g / r \pm \Delta / r$ & $M / E \pm M / r$ & سن (سال) \\
\hline .1 .97 &.$/ \ldots 9^{*}$ & 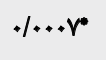 &.$\circ .8^{*}$ & $\Lambda V / \mathscr{N} \pm N$ & $q F / N F \pm N \Delta$ & FNA $\pm N q$ & وزن بلن (كيلوكرم) \\
\hline . MAY & $* \%+$ & $.1 \%+1^{*}$ & $.1 * .1^{*}$ & $r q / \backslash \pm r / \varepsilon$ & $r+18 \pm 1 / 9$ & $Y / / \pm \pm Y / \odot$ & شاخص وزن بدن (كيلوكرم/مترمربع) \\
\hline.$\ldots 1^{\circ}$ & 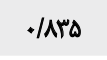 & .1 .19 & $.1 . .4$ & $\Delta / \digamma \pm \cdot / r \wedge$ & $\Delta / ৭ \pm \cdot / R T$ & $\Delta / \mp \pm \cdot / 8 \Delta$ & كلوكز (ميلىمول/ليتر) \\
\hline$* / *++f^{+*}$ & $* / Y+Y$ & $* /++\omega^{*}$ & $. /+\infty)^{*}$ & $p / q \pm 1 / 9$ & $N \perp \pm T / I$ & $\Delta / \uparrow \pm 1 / \Delta$ & انسوولين (ميكرو واحد/ ميلى ليتر) \\
\hline $.1+. r$ & $\cdot 18 \cdot V$ & $\%$ & 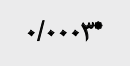 & $V / M T \pm / T t$ & $T / / r \pm+/ A T$ & $1 / T F \pm \cdot / \Delta 1$ & شاخص مقاومت أنسولين \\
\hline- & - & - &.$/ 1 \mathrm{r}$ & $\Delta V / F \pm T N$ & GNY \pm TY/D & $\Delta N \Psi \pm V / \Delta$ & (درصد) عملكرد سلول هاى بتا \\
\hline
\end{tabular}

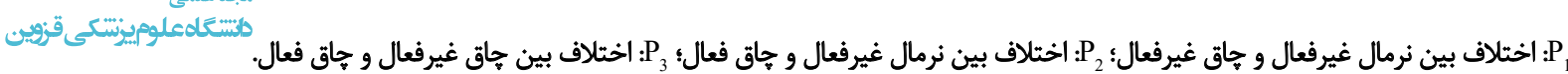

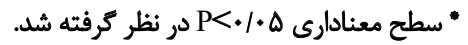




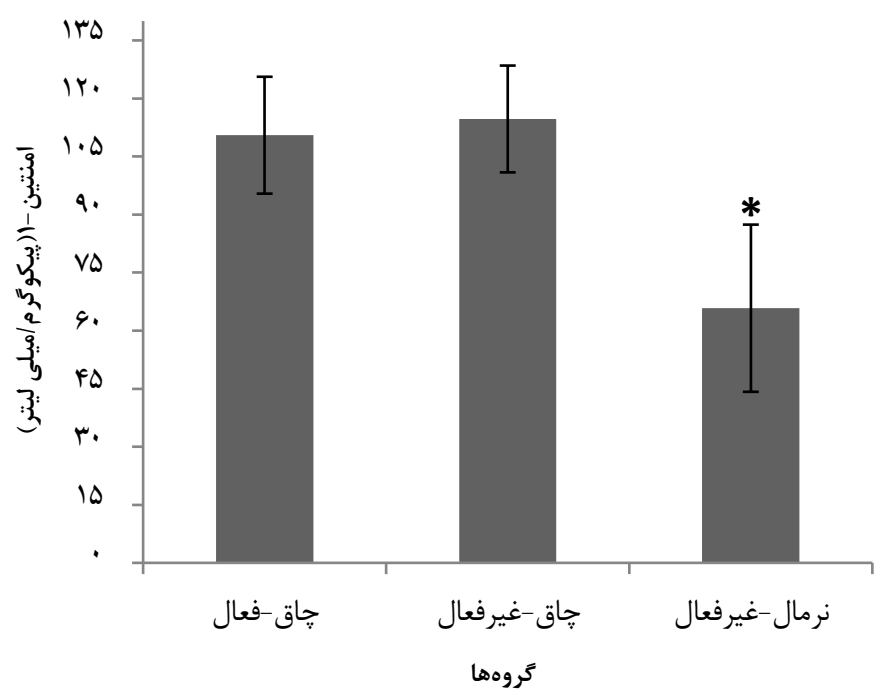

فانستعاهملوميزنتيكى قزوين

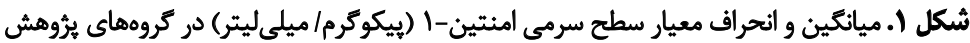

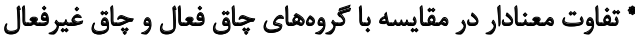

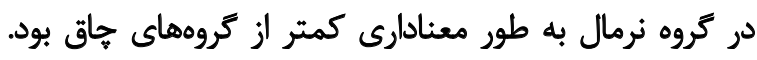

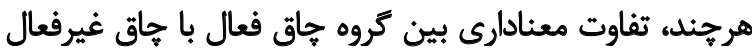

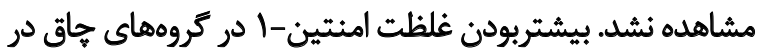

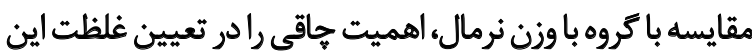

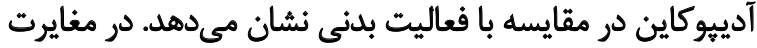

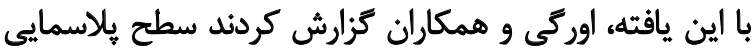

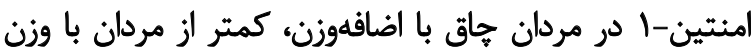

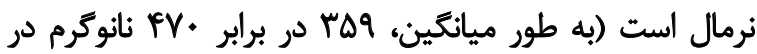

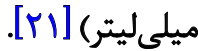
شايد يكى از دلايل اين مغايرت تفاوت در سن داوطلبان است

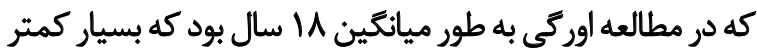

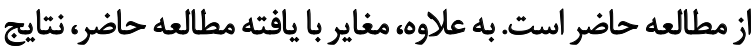

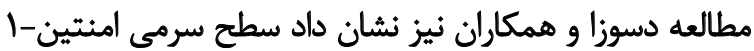

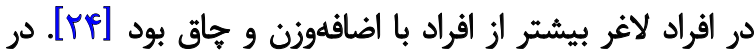

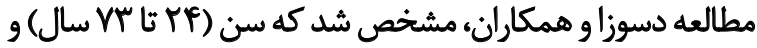

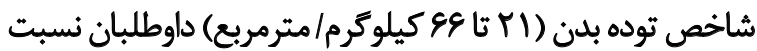

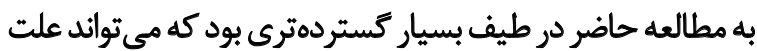

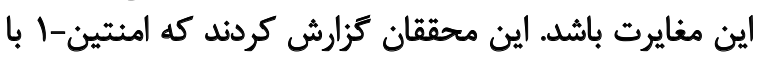

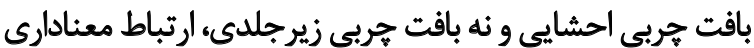

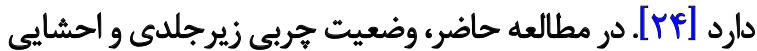

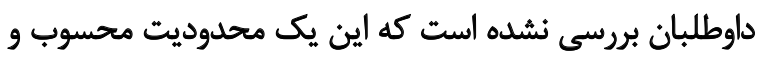
توصيه مىشود يثروهشكران در مطالعات آينده به اين نكته توجد اينه Sن

كزارش شده است كه سطح بلاسمايى امنتين-1 با شاخص

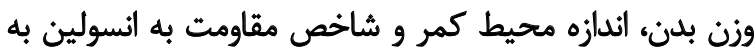

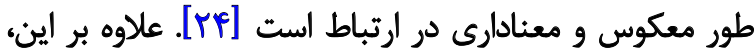

است. نتايج تحليل واريانس يكطرفه نشان داد تفاوت معنادارى

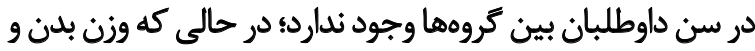

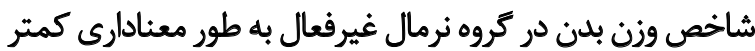

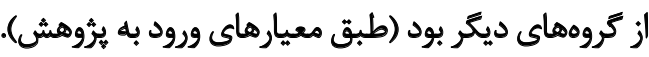
نتايج آزمون كروسكال واليس نشان داد سطح سرمى امنتين

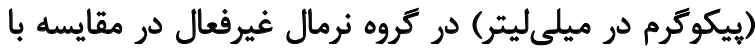

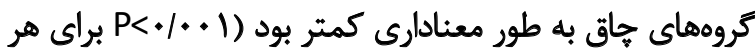

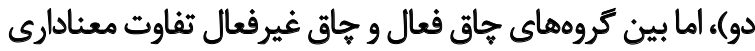

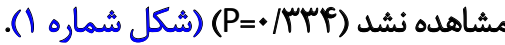
نتايج تحليل واريانس يكطرفه نشان داد غلظت انسولين (P=•/V·V)

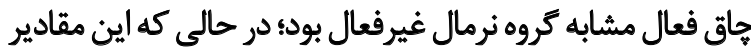

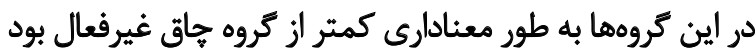

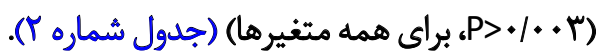
نتايج آزمون كروسكال واليس نشان داد غلظت كلوكز

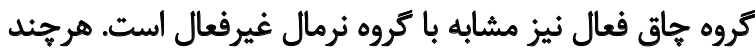

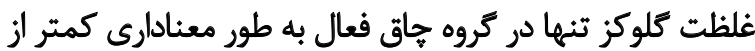

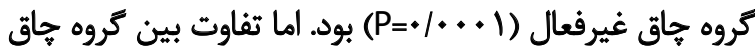

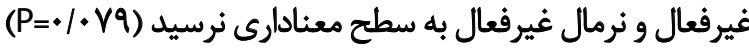

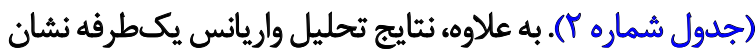

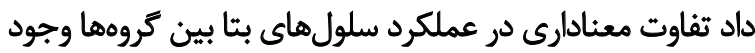

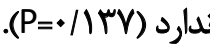

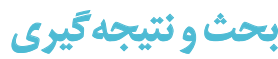
مهمثترين يافته اين مطالعه نشان داد سطح سرمى امنتين-1 
فعاليت بدنى و هاقي بود، كه ميتوانست در تفسير نتايج كمك

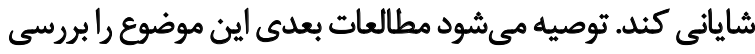
كنيند

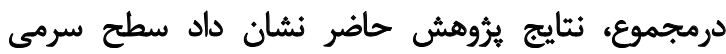

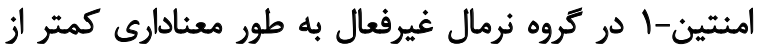

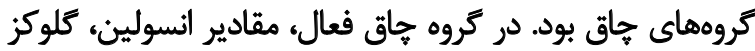

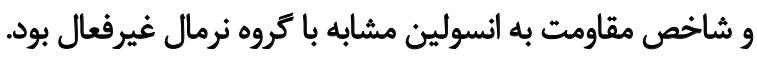

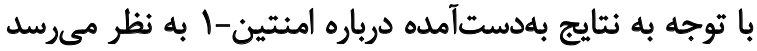

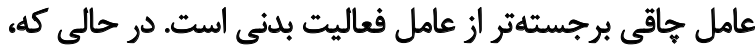

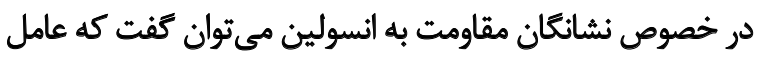

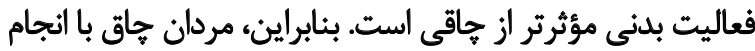

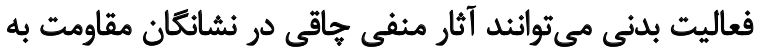

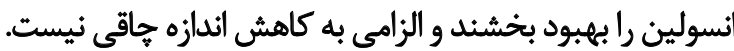

مالاحظات اخلاقي

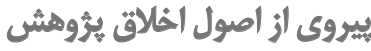

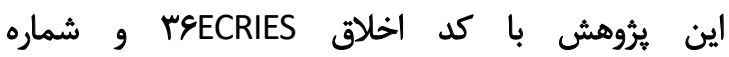
IRCT2012120411670N1 در مركز كارآزمايى بالينى ايران

به ثبت رسيد.

$$
\text { مانى مالى }
$$

اين مقاله از باياننامه كارشناسى ارشد نويسنده اول در كروه فيزيولورى ورزشى دانشكاه آزاد اسلامى واحد اليد ايلام كرفته شده

$$
\text { مشاركت نويسئد مكان }
$$

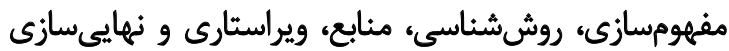

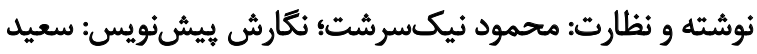

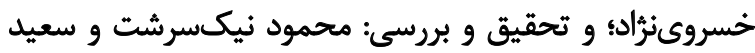

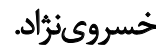

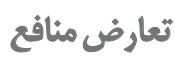

بنابر اظهار نويسندكان، اين مقاله تعارض منافع ندارد.

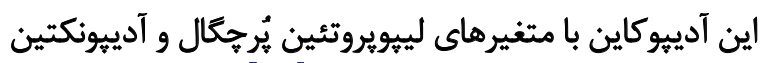

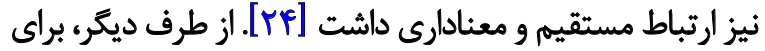

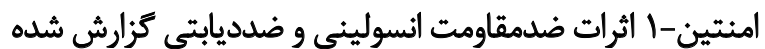

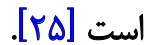

يافته ديكر مطالعه حاضر بيانكر آن است كه سطح سرميى

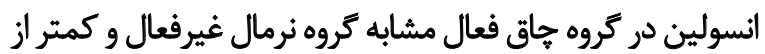

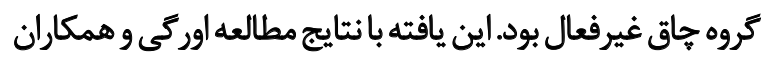

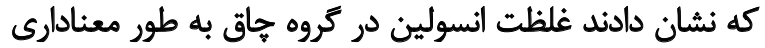

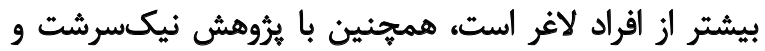

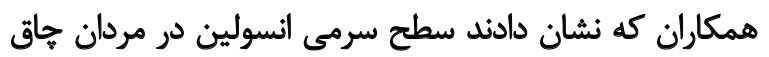

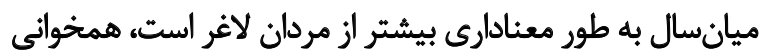

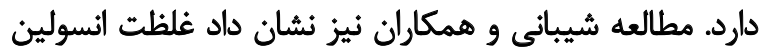

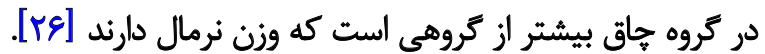

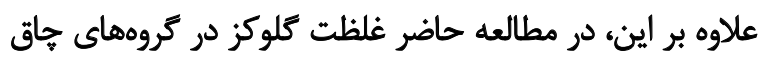

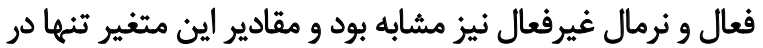

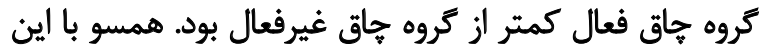

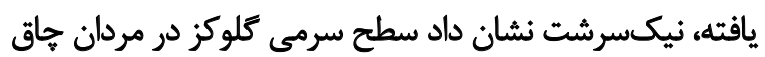

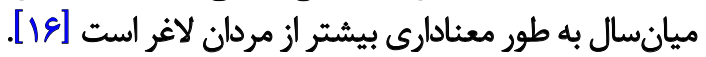
با توجه به نتايج يُروهش حاضر، كمبودن غلظت كَلوكز و و

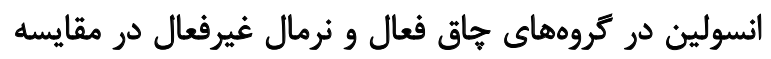

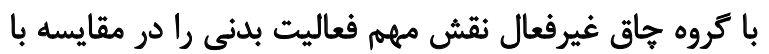

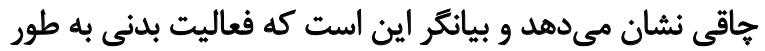

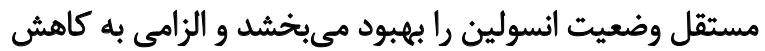
در اندازه جاقى نيست همجنين، يافته ديكر مطالعه حاضر نشان داد شاخص

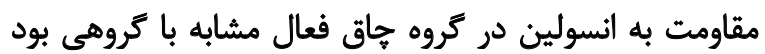

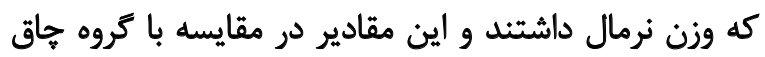

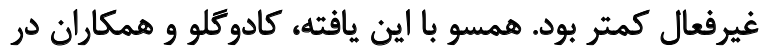

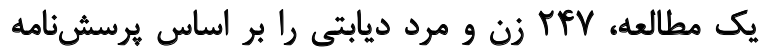

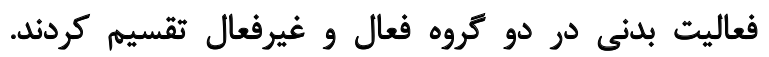

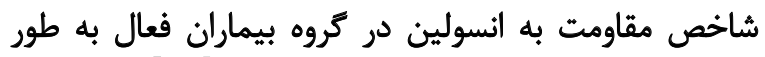
معنادارى كمتر از كروه بيماران غيرفعال بود [Trان. باتوجه به نتايج مطالعه حاضر مى توان كفت كه فعاليت بدنى در

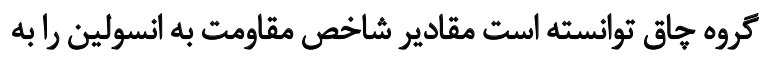

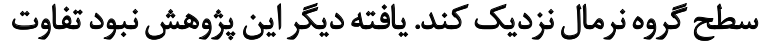

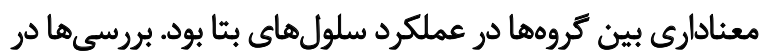

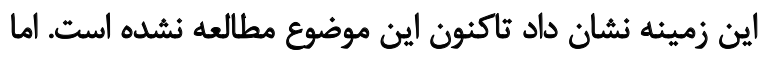

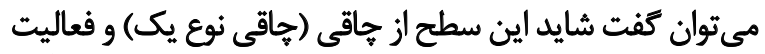

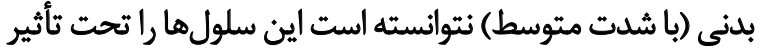

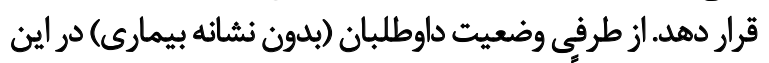

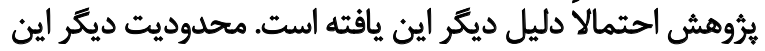

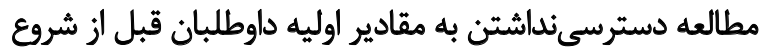




\section{References}

[1] Jialal I, Devaraj S, Kaur H, Adams-Huet B, Bremer AA. Increased chemerin and decreased omentin-1 in both adipose tissue and plasma in nascent metabolic syndrome. J Clin Endocrinol Metab. 2013; 98(3):E514-7. [DOI:10.1210/jc.2012-3673]

[2] Du Y, Ji Q, Cai L, Huang F, Lai Y, Liu Y, et al. Association between omentin-1 expression in human epicardial adipose tissue and coronary atherosclerosis. Cardiovasc Diabetol. 2016; 15:90. [DOI:10.1186/s12933-016-0406-5]

[3] Balli U, Bozkurt Dogan S, Ongoz Dede F, Sertoglu E, Keles GC. The levels of visceral adipose tissue-derived serpin, omentin-1 and tumor necrosis factor-alpha in the gingival crevicular fluid of obese patients following periodontal therapy. J Oral Sci. 2016; 58(4):465-73. [DOI:10.2334/josnusd.16-0212]

[4] Freitas Lima LC, Braga VA, do Socorro de França Silva M, Cruz JC, Sousa Santos SH, de Oliveira Monteiro MM, et al. Adipokines, diabetes and atherosclerosis: An inflammatory association. Front Physiol. 2015; 6:304. [DOI:10.3389/fphys.2015.00304] [PMID] [PMCID]

[5] Barraco GM, Luciano R, Semeraro M, Prieto-Hontoria PL, Manco M. Recently discovered adipokines and cardio-metabolic comorbidities in childhood obesity. Int J Mol Sci. 2014; 15(11):19760-76. [DOI:10.3390/ijms151119760]

[6] Zhong X, Li X, Liu F, Tan H, Shang D. Omentin inhibits TNF-alpha-induced expression of adhesion molecules in endothelial cells via ERK/NF-kappaB pathway. Biochem Biophys Res Commun. 2012; 425(2):401-6. [DOI:10.1016/j.bbrc.2012.07.110]

[7] Kazama K, Usui T, Okada M, Hara Y, Yamawaki H. Omentin plays an anti-inflammatory role through inhibition of TNFalpha-induced superoxide production in vascular smooth muscle cells. Euro J Pharmacol. 2012; 686(1-3):116-23. [DOI: 10.1016/j.ejphar.2012.04.033] [PMID]

[8] Jung SH, Park HS, Kim KS, Choi WH, Ahn CW, Kim BT, et al. Effect of weight loss on some serum cytokines in human obesity: Increase in IL-10 after weight loss. J Nutr Biochem. 2008; 19(6):371-5. [DOI:10.1016/j.jnutbio.2007.05.007] [PMID]

[9] Everett BM, Bansal S, Rifai N, Buring JE, Ridker PM. Interleukin-18 and the risk of future cardiovascular disease among initially healthy women. Atheroscler. 2009; 202(1):282-8. [DOI:10.1016/j.atherosclerosis.2008.04.015]

[10] Chan S, Cameron A, Wang Z, Venuthurupalli SK, Tan KS, Healy HG, et al. Body mass index in an Australian population with chronic kidney disease. BMC Nephrol. 2018; 19(1):209. [DOI:10.1186/s12882-018-1006-2]

[11] Willson C, Watanabe M, Tsuji-Hosokawa A, Makino A. Pulmonary vascular dysfunction in metabolic syndrome. J Physiol. 2019; 597(4):1121-41. [DOI:10.1113/JP275856]

[12] Honar doost M, Sarookhani M, Arefian E. Molecular mechanism of insulin resistance. J Qazvin Univ Med Sci. 2014; 18(5):57-64.[In Persian]

[13] Gleeson M, Bishop NC, Stensel DJ, Lindley MR, Mastana SS, Nimmo MA. The anti-inflammatory effects of exercise: Mechanisms and implications for the prevention and treat- ment of disease. Nat Rev Immunol. 2011; 11(9):607-15. [DOI:10.1038/nri3041]

[14] Nikseresht M, Sadeghifard N, Agha-Alinejad H, Ebrahim K. Inflammatory markers and adipocytokine responses to exercise training and detraining in men who are obese. J Strength Cond Res. 2014; 28(12):3399-410. [DOI:10.1519/ JSC.0000000000000553]

[15] Beavers KM, Brinkley TE, Nicklas BJ. Effect of exercise training on chronic inflammation. Clin Chim Acta. 2010; 411(11):78593. [DOI:10.1016/j.cca.2010.02.069] [PMID] [PMCID]

[16] Nikseresht M. Comparison of serum cytokine levels in men who are obese or men who are lean: Effects of nonlinear periodized resistance training and obesity. J Strength Cond Res. 2018; 32(6):1787-95. [DOI:10.1519/JSC.0000000000002039]

[17] Khoo J, Dhamodaran S, Chen DD, Yap SY, Chen RY, Tian RH. Exercise-induced weight loss is more effective than dieting for improving adipokine profile, insulin resistance, and inflammation in obese men. Int J Sport Nutr Exerc Metab. 2015; 25(6):566-75. [DOI:10.1123/ijsnem.2015-0025]

[18] Saremi A, Asghari M, Ghorbani A. Effects of aerobic training on serum omentin-1 and cardiometabolic risk factors in overweight and obese men. J Sports Sci. 2010; 28(9):993-8. [DOI:1 0.1080/02640414.2010.484070]

[19] Wilms B, Ernst B, Gerig R, Schultes B. Plasma omentin-1 levels are related to exercise performance in obese women and increase upon aerobic endurance training. Exp Clin Endocrinol Diabetes. 2015; 123(3):187-92. [DOI:10.1055/s-0034-1398504]

[20] Nikseresht M, Hafezi Ahmadi MR, Hedayati M. Detraininginduced alterations in adipokines and cardiometabolic risk factors after nonlinear periodized resistance and aerobic interval training in obese men. Appl Physiol Nutr Metabol. 2016; 41(10):1018-25. [DOI:10.1139/apnm-2015-0693] [PMID]

[21] Ouerghi N, Ben Fradj MK, Bezrati I, Feki M, Kaabachi N, Bouassida A. Effect of high-intensity interval training on plasma omentin-1 concentration in overweight/obese and normal-weight youth. Obes Facts. 2017; 10(4):323-31. [DOI:10.1159/000471882]

[22] Jackson AS, Blair SN, Mahar MT, Wier LT, Ross RM, Stuteville JE. Prediction of functional aerobic capacity without exercise testing. Med Sci Sports Exerc. 1990; 22(6):863-70. [DOI:10.1249/00005768-199012000-00021] [PMID]

[23] Haffner SM, Miettinen H, Stern MP. The homeostasis model in the San Antonio heart study. Diabetes Care. 1997; 20(7):1087-92. [DOI:10.2337/diacare.20.7.1087] [PMID]

[24] de Souza Batista CM, Yang RZ, Lee MJ, Glynn NM, Yu DZ, Pray J, et al. Omentin plasma levels and gene expression are decreased in obesity. Diabetes. 2007; 56(6):1655-61. [DOI:10.2337/db06-1506] [PMID]

[25] Watanabe T, Watanabe-Kominato K, Takahashi Y, Kojima $\mathrm{M}$, Watanabe R. Adipose tissue-derived omentin-1 function and regulation. Comprehensive Physiol. 2017; 7(3):765-81. [DOI:10.1002/cphy.c160043]

[26] Sheibani S, Hanachi P, Refahiat MA. Effect of aerobic exercise on serum concentration of apelin, TNF- $\alpha$ and insulin in obese 
women. Iran J Basic Med Sci. 2012; 15(6):1196-201. [PMID] [PMCID]

[27] Kadoglou NP, Vrabas IS, Kapelouzou A, Angelopoulou N. The association of physical activity with novel adipokines in patients with type 2 diabetes. Euro J Intern Med. 2012; 23(2):137-42. [DOI:10.1016/j.ejim.2011.10.020] 